\title{
Non-destructive Methods for Damage Assessment of Composite Sandwich Structures
}

\author{
Petr Bělský ${ }^{1, *}$, and Martin Kadlec ${ }^{1}$ \\ ${ }^{1}$ VZLU - Czech Aerospace Research Centre, Beranovych 130, 19905 Prague, Czech Republic
}

\begin{abstract}
Sandwich structures are capable of absorbing large amounts of energy under impact loads which results in high structural crashworthiness. Comparison of detection capabilities of selected C-scan NDT methods applicable for inspections of sandwich structures was performed using water-squirt, air-coupled and pitch-catch ultrasonic techniques, supplemented by laser shearography. Test results have shown that watersquirt and pitch-catch techniques are the most suitable methods for the core damage evaluation. Air-coupled method showed lower sensitive for detection of some artificial defects and impact damages in honeycomb sandwiches when unfocused transducers were used. The combination of the presented methods was able to reveal most of the defects.
\end{abstract}

\section{Introduction}

A structural sandwich is a special form of a laminated composite material which typically consists of thin stiff face sheets separated by a thicker lightweight core [1]. Thank to this advantageous arrangement, the structure can have low weight, high flexural stiffness and high buckling strength at the same time. Hence, sandwich structures are nowadays extensively used in many aerospace applications.

In terms of non-destructive testing (NDT), the structures are usually more complex which brings major challenge in some cases [2-4].

Presently, coin tap-tests or manual acousto-ultrasonic contact A-scan type methods (Pitch-Catch) are still widely used. Unfortunately, these types of inspection can be significantly affected by human factor and their output capabilities are very limited.

Utilization of automatization and C-scan methods to improve probability of detection (POD) and quality of NDT inspections is already a general trend of the last decades. C-scan imaging (2D data presentation) significantly increase quality of inspections and they allow detection of very slow and small changes in properties of the tested structures. Nowadays, $\mathrm{C}$-scans are commonly used for standard immersion ultrasonic inspections of monolithic composite structures and they can be used also for acousto-ultrasonic dry-coupled methods such as Pitch-Catch (PC). Implementation of C-scans for the technique has a great potential to improve quality of NDT of bonded joints and sandwich structures.

Contactless methods using air-coupled low-frequency (LF) or squirter-based ultrasonic transducers are also suitable for the type of tested structures. They can be successfully used

\footnotetext{
*Corresponding author: belsky@,vzlu.cz
} 
for inspections of materials which cannot be inspected using total immersion method due to possibility of damage by water ingress.

Next applicable contact-less method is laser shearography. It is a very quick and timesaving optical technique using interferometric comparison of a test object in two states, a neutral and a loaded one.

The objective of the study presented in this paper was to quantitatively compare damage size indication capabilities for a sandwich structures using various NDT techniques.

\section{Test specimen and boundary conditions}

Four reference panels with $1.3 \mathrm{~mm}$ thick CFRP skins (prepreg EHKF420-C20-45) and honeycomb (Nomex, $15 \mathrm{~mm}$ ) or foam core (Airex R82.60, $10 \mathrm{~mm}$ ) were used for the NDT activities. Two panels included artificial defects and two panels included real visible impact damage (VID) or barely visible impact damage (BVID) with impact energy 2.5 - $15 \mathrm{~J}$ using two types of impactor diameters (12.7 $\mathrm{mm}$ and $25.4 \mathrm{~mm}$ diameter). BVID impact damage was defined as impact damage with an initial dent depth less than $1.0 \mathrm{~mm}$. Impacting was performed on SUPR drop impact tower in accordance to ASTM D7136M. The drop tester was equipped with accelerometer and anti-rebound system. The data obtained from the accelerometer was used for the complete analysis of the behavior of the specimens using information about the real absorbed energy. Dent depth was measured with indicator gauge immediately after impact and after relaxing (30 minutes). Fig.1 shows honeycomb inspection reference panel IRP1 with artificial defects. Design of the panel IRP2 with foam core was very similar but potted core cells were replaced with $3 \mathrm{~mm}$ deep flat-bottom holes. Impacted specimens had the same dimensions as IRPs panels. Impacts were performed in two rows with $80 \mathrm{~mm}$ spacing.

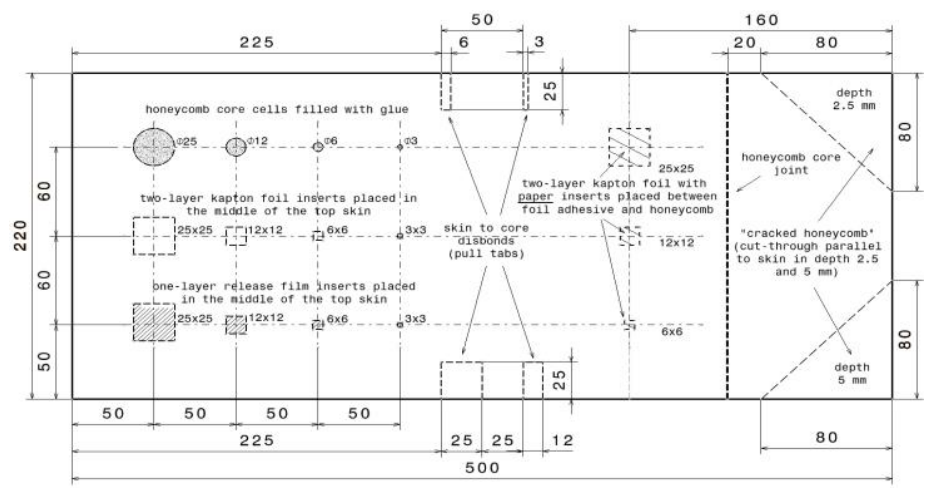

Fig. 1. Inspection reference panel IRP1 with artificial defects

\section{Experimental methods}

\subsection{Water-squirt technique}

Initially, the panels were scanned by pulse-echo (PE) and through transmission (TT) watersquirt techniques in which the ultrasound was coupled by a narrow water jet. Universal squirter nozzles made by $3 \mathrm{D}$ printing and single element pencil case type unfocused transducers Olympus V323-N-SU 2.25 MHZ (TT) and V310-N-SU 5 MHz (PE) were used (Fig. 2a). Expected high sensitivity and resolution was confirmed but the C-scans of panels with foam core could not be done because a suitable pencil case type probe with frequency below $1 \mathrm{MHz}$ was not available. 


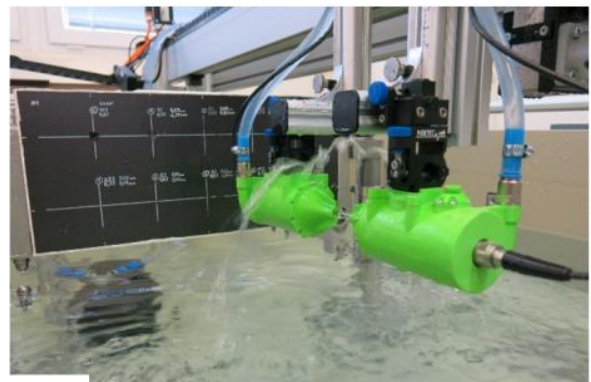

a)

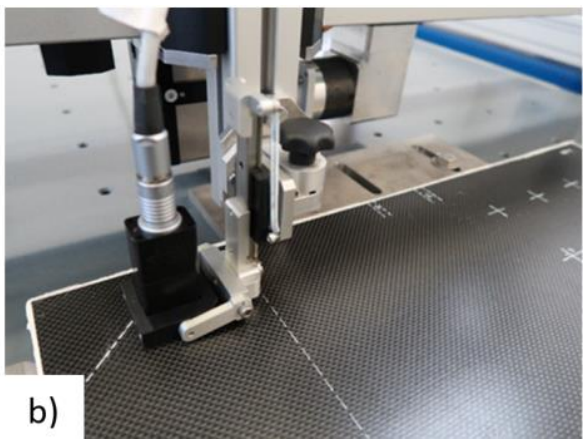

b)

Fig. 2. Ultrasonic methods: a) TT water-squirt technique, b) PC method utilizing ECA module

\subsection{Pitch-Catch}

Next scans were performed by pitch-catch method using an innovative Omniscan Bond Tester utilizing standard ECA module. Pitch-catch method employs a dual-element, pointcontact, dry-coupled ultrasonic probe. One element transmits a burst of acoustic energy into the test part and a separate element receives the sound propagated across the test piece between the two probe tips. The bond condition beneath the two tips can be determined and measured data is visualized by means of amplitude or phase C-scans. The method allows far-side defect detection.

Broadband pitch-catch probe SPO-5629-PHV with measuring tips spacing $12.7 \mathrm{~mm}$ was used (Fig. 2b). C-scans were performed for the test frequencies of $8-42 \mathrm{kHz}$ during the optimalization phase. The best results were obtained for frequencies of $11-13 \mathrm{kHz}$ and probe drive $4 \mathrm{~V}$.

\subsection{Air-coupled ultrasound}

Air-coupled LF testing method working with frequencies below $1 \mathrm{MHz}$ is suitable for contactless inspections of high ultrasonic attenuation materials which cannot come in contact with water. Special dual element transducers in two separate housings working separately as transmitter and receiver are required for most applications (Fig. 3a). Because of this, standard pulse-echo testing is not feasible.

LF system STARMAN DIO 2000, analysis software C-Scan and unfocused $120 \mathrm{kHz}$ probes PLN-19-012 with nominal element size of $19 \mathrm{~mm}$ were used for the experiments.
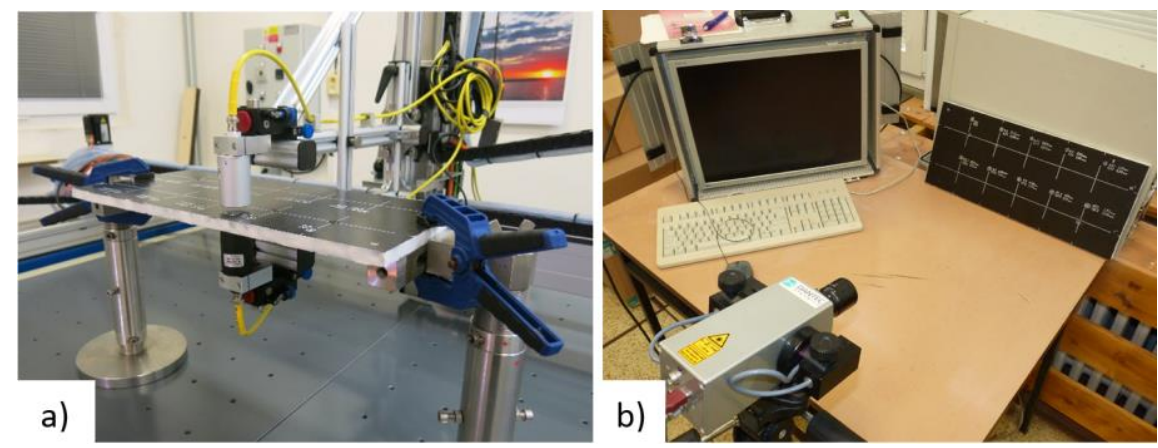

Fig. 3. Compared Contactless NDT methods: a) Air-coupled ultrasound, b) Laser shearography 


\subsection{Laser Shearography}

Laser Shearography (LS) is a large area optical inspection system that uses laser light to detect very slight surface deformations due to subsurface discontinuities $[5,6]$. Shearography uses the interference of monochromatic laser light to detect surface displacements in the order of nanometres (Fig. 3b). It is an 'Active' NDT Method; it measures the materials reaction to an applied stress. The reference panels were loaded by heat (500 W from $20 \mathrm{~cm}$ distance during 5 seconds). Horizontal shear was used. Wrapped phase image was evaluated.

\section{Results and discussion}

Water-squirt ultrasonic technique, Air-coupled ultrasound, Pitch-Catch method and Laser Shearography were used to detect defects in four composite sandwich panels with honeycomb or foam core. Two panels included different types of artificial defects and two panels included real BVID and VID impact damages.

All amplitude $\mathrm{C}$-scans performed with the PE and TT water-squirt techniques showed excellent resolution and sensitivity of the methods. However utilization of high frequencies is limited due to high attenuation of the structures. Frequencies below $1 \mathrm{MHz}$ are needed for TT inspections of sandwich structures with foam core.

The Pitch-Catch method also proved to be very sensitive but its resolution is limited to defects greater than $12 \times 12 \mathrm{~mm}$ because of the tip spacing on the PC probe $(12.7 \mathrm{~mm})$. The method is also not suitable for inspections of very rough or damaged surfaces. Measuring tips of PC probe can be damaged in case of some opened surface defects. On the other hand the method has high penetration and thanks to that far-side defects can be readily detected.

Experiments with air-coupled low-frequency method have shown that the method can be less sensitive for detection of some types of artificial defects in honeycomb core sandwich. All one-layer and most of two-layer kapton inserts with dimensions smaller than $25 \times 25 \mathrm{~mm}$ were not reliably detected (see Fig. $4 \mathrm{c}$ ). On the other side, the detection capability of the defects was significantly better for foam-core sandwiches. All defects in IRP2 and F1 panels were detected (see Fig. 5c and Fig. 7c). One reason of worse detection capability of defects in honeycomb sandwiches may be that unfocused transducers were used. Utilization of focused transducers or apodization of the receiving transducer could significantly increase resolution of the method [7].

Laser shearography was able to detect most of the artificial and real impact defects but its sensitivity was significantly worse in case of honeycomb sandwiches. Sizing of impact damages was not reliable. The method can detect defects and impact damages above all in the skins. It is not able to detect some fine damage of the core by the method.

Selected final C-scans and more detailed description of the results are given in the following subchapters.

\subsection{Artificial defects}

Fig. 4 shows C-scans of inspection reference panel IRP1 with artificial defects. All defects in the test specimen were possible to detect by the combination of PE and TT water-squirt methods. TT technique was insensitive to detection of thin one-layer inserts (foreign bodies) in the skin.

Two types of C-scans were used for Pitch-Catch method: the amplitude C-scans displaying colour variation based on the amplitude of the signal (ideal for disbond and potted core detection) and the phase $\mathrm{C}$-scans using a $0^{\circ}$ to $360^{\circ}$ colour palette to display changes in the phase angle (ideal for delamination and foreign bodies detection in skins). 
All disbonds, potted core cells and core damages were very well evident in the amplitude C-scan obtained at optimized frequency $13 \mathrm{kHz}$ (see Fig. 4b). Two-layer inserts (delaminations) greater than $12 \times 12 \mathrm{~mm}$ were well evident in the phase $\mathrm{C}$-scan obtained at $42 \mathrm{kHz}$. No one-layer inserts were detected.

Results obtained by air-coupled LF method and laser shearography indicated that these methods were not able to reliably detect two-layer inserts smaller than $25 \times 25 \mathrm{~mm}$ and all one-layer inserts in honeycomb sandwich structures.

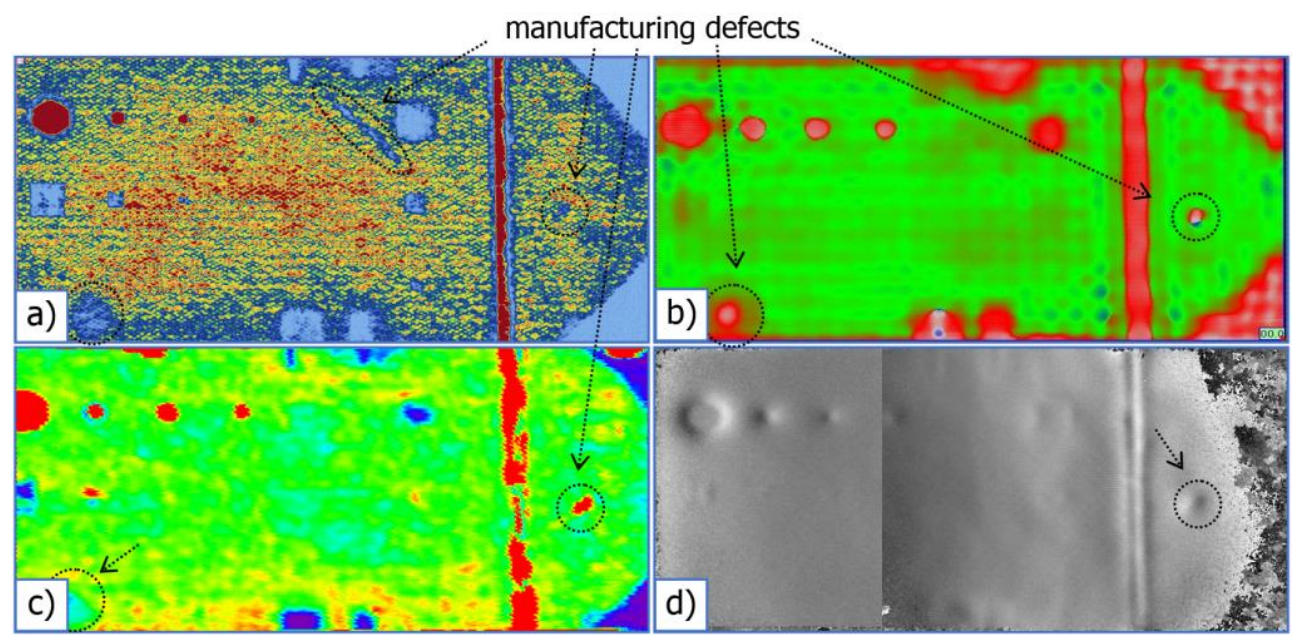

Fig. 4. C-scans of IRP1 (Nomex honeycomb core) performed using the methods: a) TT water-squirt technique, b) Pitch-Catch, c) TT Air-coupled ultrasound, d) Laser Shearography

Fig. 5 shows C-scans of Airex foam core sandwich panel IRP2. Unfortunately, the TT Cscan by water-squirt technique could not be performed because suitable transducers with frequency under $1 \mathrm{MHz}$ were not available. That is why only PE C-scan was performed by the method (see Fig. 4a). The amplitude PE C-scan is very interesting because all artificial defects were detected except foam core defects in the right-hand corners of IRP2.

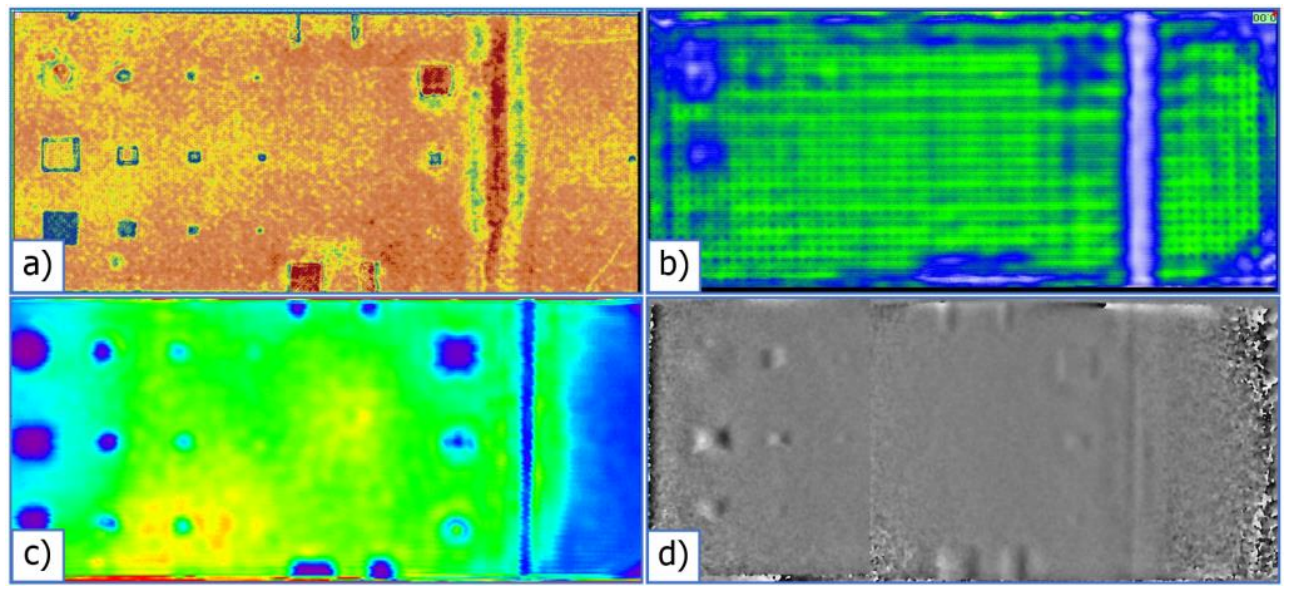

Fig. 5. C-scans of IRP2 (Airex foam core) performed using the methods: a) PE water-squirt technique (top skin), b) Pitch-Catch, c) TT Air-coupled ultrasound, d) Laser Shearography

Also, air-coupled LF method and laser shearography were able to reliably detect all defects up to $6 \times 6 \mathrm{~mm}$. The defects $3 \times 3 \mathrm{~mm}$ were not reliably detected. 
Detection capability of Pitch-Catch was significantly worse for the test panel in comparison with above mentioned methods. It was not able to reliably detect defects smaller than $25 \times 25 \mathrm{~mm}$.

\subsection{Impact damage}

The main objective of the next part of the study was to quantitatively compare real impact damage size indication in sandwich structures using various NDT techniques. Two sandwich panels H1 and F1 with Nomex honeycomb and Airex R82.60 foam core respectively were impacted on SUPR drop impact tower. Ten impacts with different impact energy were applied on each test panel. BVID damages were achieved for total impact energy less than $5 \mathrm{~J}$ in case of 0.5 " impactor and $7 \mathrm{~J}$ in case of 1 " impactor.

Final C-scans of the impacted panels are shown in Fig. 6 and Fig. 7. All compared NDT methods were able to detect all impact damages, but they differed in sizing of damages.

Several manufacturing defects were detected at the edge of the honeycomb sandwich panel H1 by TT water-squirt and PC methods (see Fig. 6).

Water-squirt technique has proved to be very sensitive to any damage of honeycomb core. This finding confirms some conclusions of IRP1 reference panel inspection. Comparison of C-scans obtained by TT and PE water-squirt technique is very interesting. While the PE C-scan shows damage only in the top skin, TT C-scan shows overall damage of impacted sandwich. The difference in the indicated damage size is significant in this case. It shows that the honeycomb core is sensitive to impacts and water-squirt method is able to detect even very small damages of the core.

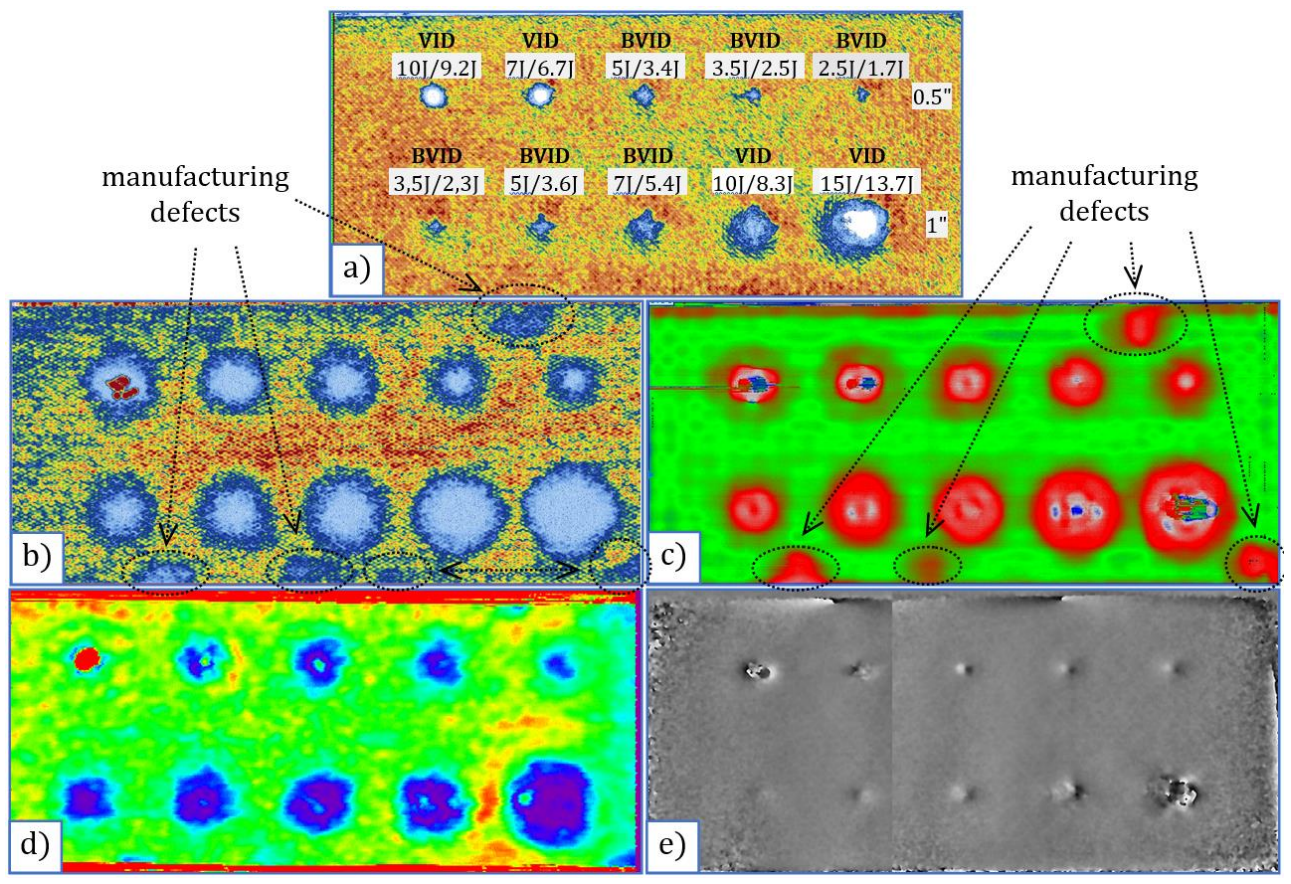

Fig. 6. C-scans of impacted H1 test panel (Nomex honeycomb core) performed using the methods: a) PE water-squirt technique (C-scan of upper skin), b) TT water-squirt technique, c) Pitch-Catch, d) TT Air-coupled ultrasound, e) Laser Shearography 
Similar comparison of capabilities of NDT methods was performed also for impacted foam core sandwich. Fig. 7 shows final C-scans of test panel F1 with Airex R82.60 foam core.

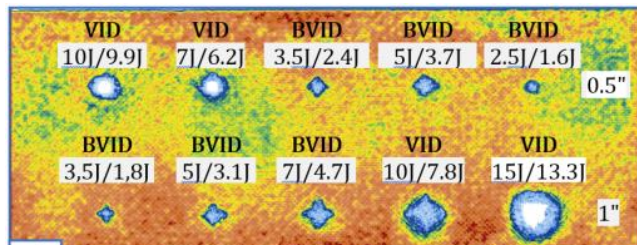

a)

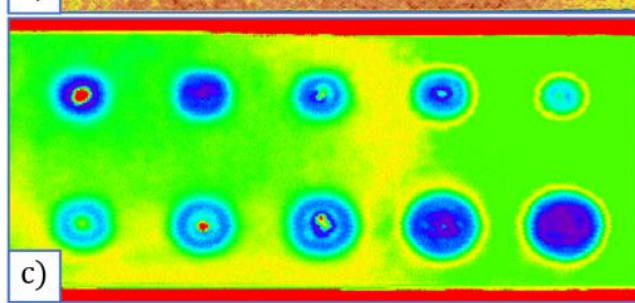

b)

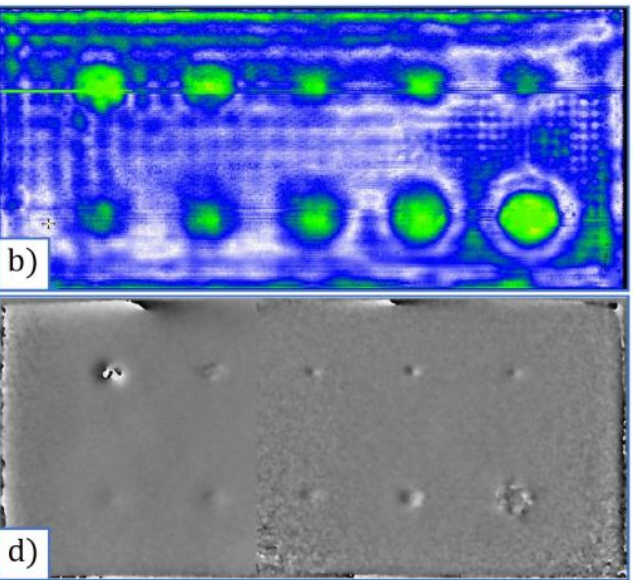

Fig. 7. C-scans of impacted F1 test panel (Airex foam core) performed using the methods: a) PE water-squirt technique, b) Pitch-Catch, c) TT Air-coupled ultrasound, d) Laser Shearography.

All impact damage indications in C-scans obtained by four NDT methods were measured in horizontal and vertical direction. The average values for each indication are presented in graphs in Fig. 8. Apparently, TT water-squirt and Pitch-Catch methods were the most suitable methods for the core damage evaluation. Laser shearography and PE water-squirt methods detected only the skin damage.
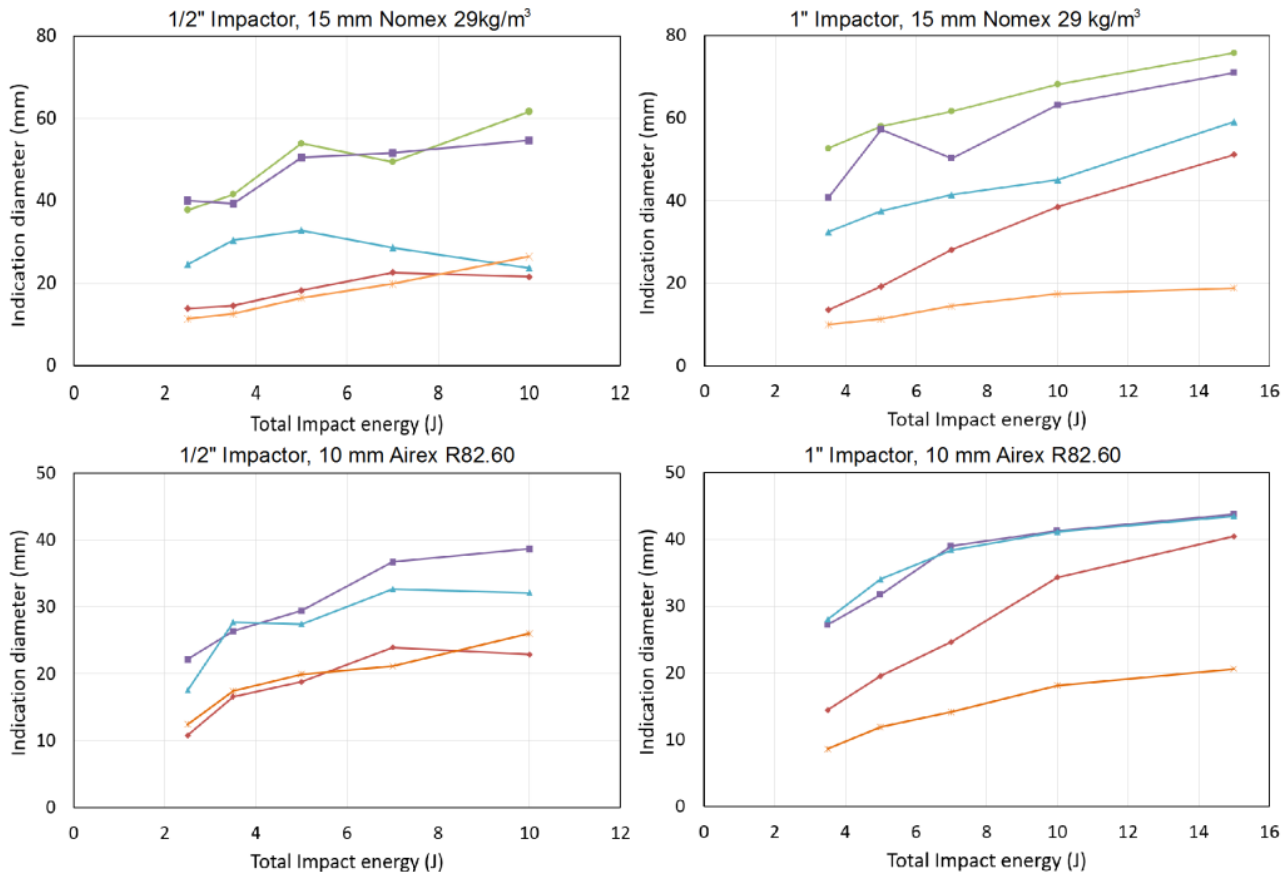

$\rightarrow$ Squirter tech. PE $\rightarrow$ Squirter tech. TT $\rightarrow$-Pitch-Catch $₫$ Air-coupled UT $\because$ Shearography

Fig. 8. Indication diameters for various NDT methods related to the total impact energy. 


\section{Conclusions}

Comparison of detection capabilities of four C-scan NDT methods applicable for inspections of sandwich structures was performed in the study.

Four reference panels with $1.3 \mathrm{~mm}$ thick CFRP skins (prepreg EHKF420-C20-45) and honeycomb (Nomex, $15 \mathrm{~mm}$ ) or foam core (Airex R82.60, $10 \mathrm{~mm}$ ) were used for the NDT activities. Two panels included different types of artificial defects imitating delaminations and foreign objects in the skin, unbonded skin-core joint, potted core cells, cracked core, etc. Next two panels were used for impact behavior study of sandwich structures made of above mentioned materials. Ten impacts with different impact energy were applied on each test panel and BVID and VID damages were induced.

Water-squirt ultrasonic technique, Air-coupled ultrasound, Pitch-Catch method and Laser Shearography were used for detection of artificial defects and real impact damages in above mentioned sandwich panels. Water-squirt technique has proved to be extremely sensitive to any damage of the honeycomb core. Comparison of C-scans obtained by TT and PE water-squirt technique for honeycomb sandwich panels showed significant differences in the indicated damage size. It shows that the honeycomb core is sensitive to impacts and TT water-squirt method is able to detect even very minor damages of the core.

The Pitch-Catch method also proved to be very sensitive but its resolution is limited to defects greater than $12 \times 12 \mathrm{~mm}$ because of the tip spacing on the PC probe $(12.7 \mathrm{~mm})$. The method is also not suitable for inspections of very rough or damaged surfaces.

Air-coupled low frequency method showed a little lower sensitivity for detection of some artificial defects and impact damages in honeycomb core type sandwiches when unfocused transducers were used. Utilization of focused transducers or apodization of the receiver could significantly increase resolution of the method. Sensitivity of the method was fully comparable with Pitch-Catch method in case of foam core type sandwiches. It was able to well detect all defects in the foam core test panels.

Laser shearography was able to detect most of defects in the panels but it had lower sensitivity and resolution in the case of honeycomb core type sandwiches. The method is able to detect impact damages only in the skins.

To conclude, the combination of the presented methods was able to reveal all of the artificial defects and real impact damages. The results obtained in the study will be used in frame of research activities focused on structural health monitoring of impacted sandwich structures using guided waves.

\section{Acknowledgements}

These R\&D works were realized within the Institutional support of the Ministry of Industry and Trade of Czech Republic for the development of research organization.

\section{References}

1. A. Herrmann, P. Zahlen, I. Zuardy, Proc. Int. Conf. Sandwich Struct., 7, 13-26 (2005)

2. M. Kadlec, R Růžek, Appl. Compos. Mater. 19, 393-407 (2012)

3. J.H. Heida, D.J.Platenkamp, WCNDT 2012, 3, 2324-2337 (2013)

4. N.Takeda, S. Minakuchi, Y. Okabe, JSMME, 1, 3-17 (2007)

5. J.W. Newman, Materials Evaluation, 63, 746-750 (2005)

6. P. CAWLEY, ECNDT 2006 - Mo.2.6.1, (2006)

7. J.Peters, V.Kommareddy, Z.Liu, D.Fei, AIPC Proceedings, 657, 973-980 (2003) 